\title{
A mass obstructing mechanical prosthetic heart valve: thrombus or pannus?
}

\author{
Kunal Mahajan, Sanjeev Asotra, Prakash Negi, Prince Kumar Paul
}

Department of Cardiology, Indira Gandhi Medical College, Shimla, India

Correspondence to Dr Kunal Mahajan, kunalmahajan442@gmail.com

Accepted 22 May 2017
CrossMark

To cite: Mahajan $\mathrm{K}$, Asotra $\mathrm{S}$, Negi P, et al. BMJ Case Rep Published Online First: [please include Day Month Year]. doi:10.1136/bcr-2017219573

\section{DESCRIPTION}

Mechanical prosthetic valve obstruction carries a significant morbidity and mortality, if not treated urgently. Most notable causes of an acquired obstruction include thrombosis and pannus formation. ${ }^{1}$ It is critically important to exclude isolated pannus, since thrombolysis is an effective and rapid treatment option in case of a thrombus, while it is contraindicated in pannus. Published guidelines provide no diagnostic strategy to differentiate them. ${ }^{2}$ Transesophageal echocardiogram (TEE) is indispensable for assessing the leaflet motion and the nature and extent of the obstructing mass. However, in resource-poor settings, where TEE is not always available, therapy is based on transthoracic echocardiography and fluoroscopic findings.

A 50-year-old woman presented with complaints of worsening dyspnoea associated with orthopnoea

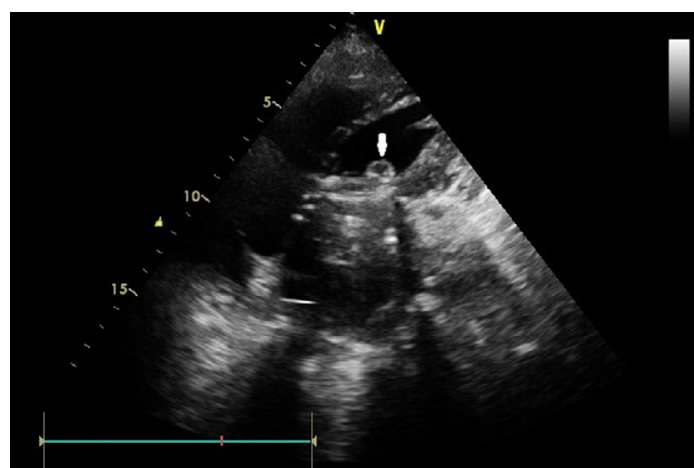

Figure 2 Transthoracic echocardiogram four-chamber view showing a small rounded mass (arrowhead) with echogenic borders and echolucent central portion attached on the ventricular side of the mitral valve prosthesis.

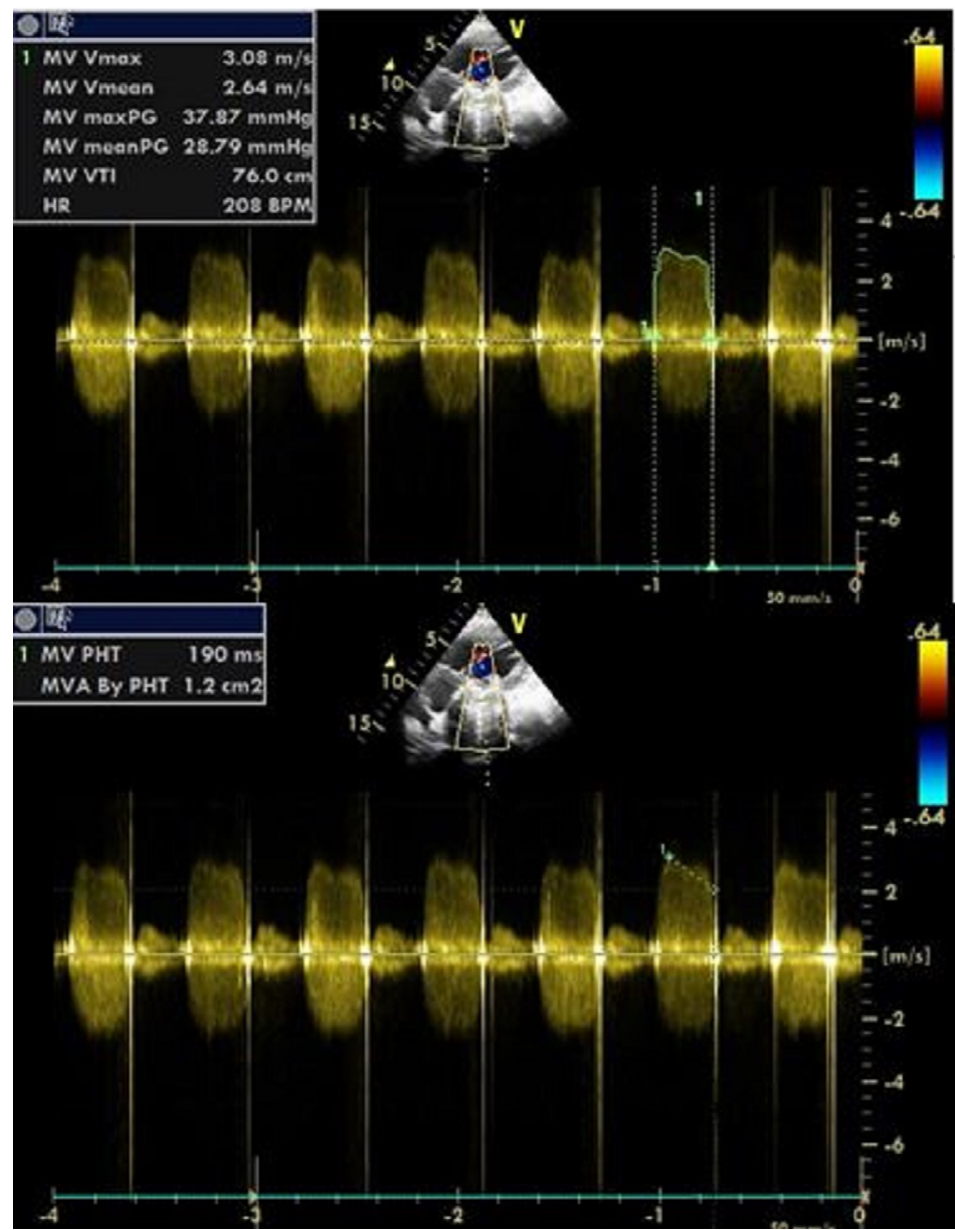

Figure 1 Demonstration of reduced valve area and the increased gradients across the prosthetic valve using continuous-wave Doppler echocardiography. 


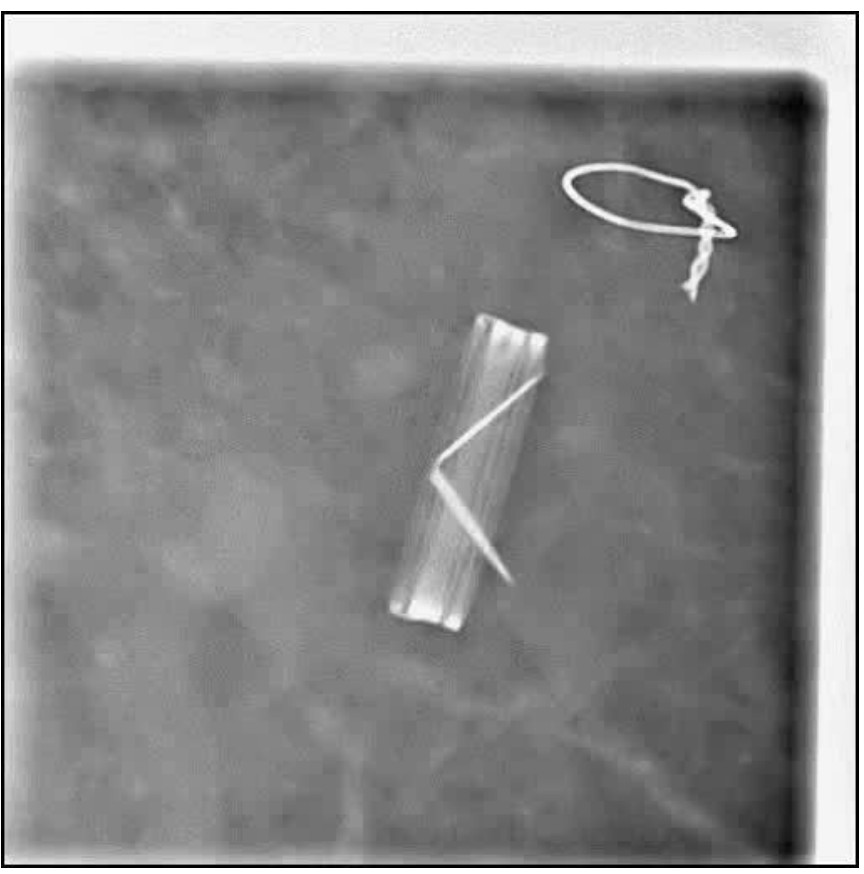

Video 1 Fluoroscopy showing restricted opening of both the leaflets. Note that one of the leaflets appears totally blocked in semi-open position, while the other shows mildly restricted opening.

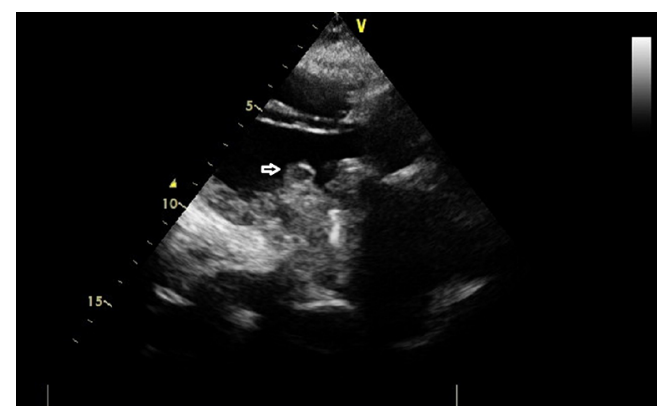

Figure 3 Transthoracic echocardiogram parasternal long-axis view, showing the thrombus (arrowhead) attached on ventricular side of mechanical prosthetic mitral valve. Note the acoustic shadow of the metallic prosthesis, obscuring the visualisation of leaflets and extent of attachment of the thrombus.

for 3 days. She had undergone a mitral valve replacement with a mechanical bi-leaflet prosthesis 6 months prior for severe rheumatic mitral regurgitation, following which she was asymptomatic. Examination revealed blood pressure of $90 / 64 \mathrm{~mm} \mathrm{Hg}$ and sinus tachycardia with heart rate of $120 / \mathrm{min}$ along with decreased intensity of the closing click on auscultation. She had a suboptimal international normalised ratio (INR) of 1.36. Fluoroscopy showed severely restricted leaflet opening (video 1). Transthoracic echocardiogram revealed significantly increased gradient across the mitral valve prosthesis (figure 1). It also revealed a small rounded $8 \times 10 \mathrm{~mm}$, minimally mobile, echogenic mass with central echolucent area, attached to the prosthetic ring on the ventricular side (figures 2

Figure 4 Image showing complete resolution of the thrombus after thrombolysis with streptokinase.

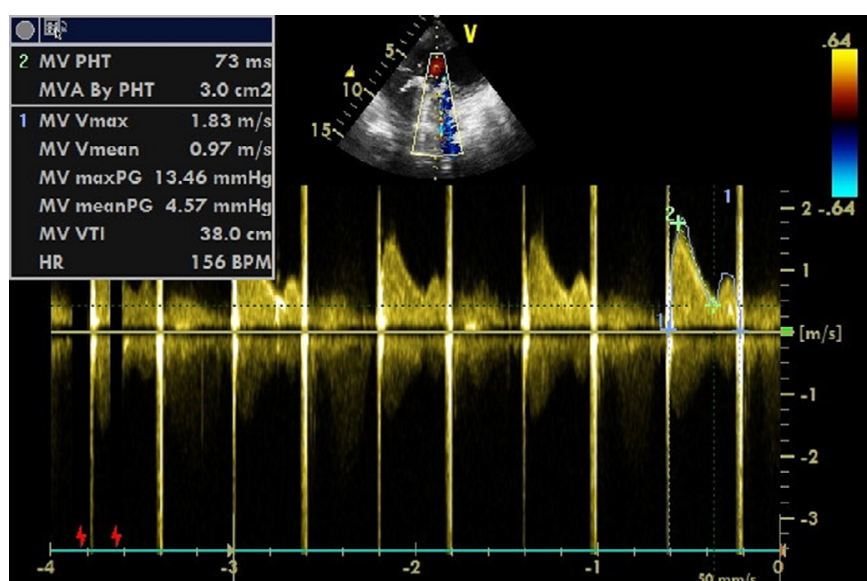

Figure 5 Post-thrombolysis echocardiogram showing decreased gradients and normal valve area.

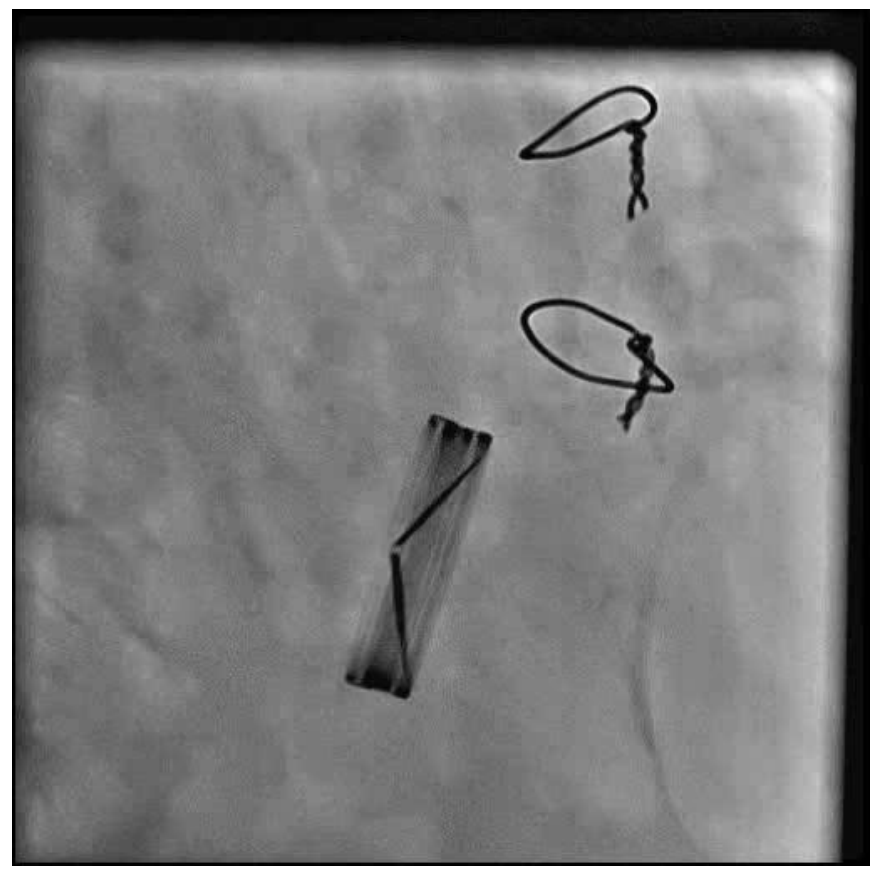

Video 2 Post-thrombolysis fluoroscopy showing significant improvement in the motion of the leaflets.

and 3). Extent of its dimensions and leaflet attachment could not be assessed, especially because of the unavailability of TEE at that time.

Presentation within 6 months of implantation with acute onset symptoms, low INR and low echodensity on echocardiogram suggested the diagnosis of thrombus. Thrombolysis with streptokinase was done over 24 hours which resulted in marked relief in symptoms. Repeat echocardiogram showed no residual thrombus and normalised gradient across the prosthetic valve (figures 4 and 5). Normal leaflet excursion was confirmed on fluoroscopy (video 2).

Contributors KM and SA made the diagnosis and performed the complete investigations and were involved in the management of the patient. KM wrote the manuscript. PN performed the literature search. PN and PKP corrected the manuscript and gave conceptual advice. All authors read and approved the final version of the manuscript.

Competing interests None declared.

Patient consent Obtained. 


\section{Learning points}

- The acquired causes of mechanical prosthetic valve obstruction include thrombus, pannus formation and rarely endocarditis.

- While the absence of fever, systemic features of endocarditis and negative blood cultures can easily exclude endocarditis, differentiation of thrombus from pannus requires careful assessment of history, INR and the echocardiography features.

- Tissue overgrowth responsible for pannus formation usually occurs over a period of 6 months or more, and therefore, long interval after valve insertion is required for pannus to cause valve dysfunction. Importantly, obstruction by a pannus does not cause acute symptoms.

- Acute presentation, subtherapeutic INR and low echodensity of the obstructing mass are some of the features that favour the diagnosis of a thrombus.

- Restricted leaflet opening on fluoroscopy can be present in both thrombus and pannus. Its absence makes the diagnosis of thrombus very unlikely. However, pannus can cause obstruction without restricting leaflet opening.

visic casereporis. Drril.com tor more arucies IIKe unis ana to vecome a renow
Provenance and peer review Not commissioned; externally peer reviewed.

(c) BMJ Publishing Group Ltd (unless otherwise stated in the text of the article) 2017. All rights reserved. No commercial use is permitted unless otherwise expressly granted.

\section{REFERENCES}

1 Habets J, Budde RP, Symersky P, et al. Diagnostic evaluation of left-sided prosthetic heart valve dysfunction. Nat Rev Cardiol 2011;8:466-78.

2 Vahanian A, Alfieri O, Andreotti F, et al. Guidelines on the management of valvular heart disease (version 2012): The Joint Task Force on the management of valvular heart disease of the European Society of Cardiology (ESC) and the European Association for Cardio-Thoracic Surgery (EACTS). Eur Heart J 2012;33:2451-96.

e any of this content visit

ithout any further permission. m 